\title{
Subthreshold Laser Treatment for Serous Retinal Detachment Associated with Tilted Disc Syndrome
}

\author{
Yuko Minowa Kishiko Ohkoshi Yoko Ozawa \\ Ophthalmology Department, St. Luke's International Hospital, Tokyo, Japan
}

\section{Keywords}

Endpoint laser · Micropulse laser · Subthreshold laser · Tilted disc syndrome

\begin{abstract}
Persistent serous retinal detachment (SRD) is a common complication of tilted disc syndrome (TDS). The purpose of this study is to evaluate the efficacy of subthreshold laser photocoagulation for SRD associated with TDS. This retrospective, single-center study included 5 eyes of 5 patients with TDS-associated SRD treated by subthreshold laser treatment. SRD was completely absorbed in 4 eyes within 4 months after initial treatment. However, it recurred in 2 eyes; one required additional laser treatment and one showed spontaneous resolution. Eventually, all 4 eyes showed complete SRD resorption. The mean visual acuities at enrollment and 1 and 3 months showed no significant differences. The mean central macular thickness showed a significant decrease at 3 months. Two eyes showed changes in fundus autofluorescence findings at the laser ablation site. However, there were no instances of laser scotoma and no laser-induced retinal scarring on color fundus photography performed the end of treatment. In conclusion, subthreshold laser photocoagulation is an effective treatment for SRD associated with TDS. Thus, the clinical indications of subthreshold laser photocoagulation may be extended to SRD in patients with TDS.
\end{abstract}

\section{Introduction}

Tilted disc syndrome (TDS) is a congenital anomaly characterized by an inferonasally "tilting" optic disc with associated inferior staphyloma. Macular complications of TDS include polypoidal choroidal vasculopathy, classic choroidal neovascularization, focal serous retinal

\section{Karger!}


detachment (SRD), and atrophy of the retinal pigment epithelium (RPE) [1]. Among these complications, focal SRD is the most common finding (41\%) [1]. Focal SRD results from scleral thickening and choroid thinning that leads to abnormal choroidal circulation and breakdown of the blood-retina barrier [2]. Although persistent SRD is a major cause of irreversible visual acuity deterioration, no treatment has been reported to be effective for this condition. Although intravitreal bevacizumab and photodynamic therapy (PDT) have been used as treatments for TDS $[3,4]$, they have not been effective.

Of late, subthreshold laser photocoagulation has been used to treat various macular diseases such as diabetic macular edema [5] and central serous retinopathy [6]. Subthreshold micropulse laser photocoagulation is a unique laser technique to minimize laser damage by the application of a train of short-duration laser pulses, chopping the laser beam into an envelope of repetitive short pulses [5]. Endpoint Management (Topcon Corp., Santa Clara, CA, USA) is a subthreshold laser system that calculates parameters to determine the amount of laser-induced tissue damage. These modalities are designed to provide selective photocoagulation of the RPE while having minimal effects on the sensory retina and choroid. Recent literature has revealed the efficacy of a minimally invasive laser system for diabetic macular edema [5] and central serous retinopathy [6]. However, the efficacy of these methods for SRD in patients with TDS has not yet been investigated. Accordingly, the aim of the present study was to evaluate the efficacy of a subthreshold laser system to treat TDS-associated SRD.

\section{Case Report/Case Presentation}

This retrospective study followed the tenets of the Declaration of Helsinki. The Institutional Review Board at St Luke's International Hospital approved this study (No. 17-R114). Five eyes of 5 consecutive patients with TDS-associated persistent SRD ( $>1$ year) treated between January 2011 and September 2016 at St Luke's International Hospital were enrolled. TDS was diagnosed by the characteristic inferonasal tilting of the oval optic disc, with a congenital inferonasal crescent and an inferior staphyloma at the fovea, using optical coherence tomography (OCT). TDS patients with SRD persisting for at least 3 months after onset were enrolled.

A comprehensive ophthalmic examination, including best-corrected visual acuity measurement, color fundus photography, OCT, and fundus autofluorescence (FAF), was performed prior to and 1 and 3 months after treatment. Measurements were repeated when deemed clinically necessary. Fluorescein angiography and indocyanine green angiography were conducted at enrollment to rule out the presence of choroidal neovascular membrane associated with other diseases. Visual acuity was determined using the Snellen chart, and logarithms of the minimal angle of resolution (logMAR) values were calculated for statistical analysis. The central macular thickness was measured using the Cirrus-OCT system (CarlZeiss Meditec, Dublin, CA, USA), and FAF was performed using a Heidelberg Spectralis device (Heidelberg Engineering, Heidelberg, Germany). The development of laser-induced scotoma was investigated at each follow-up visit using Amsler grid screening.

Patients underwent subthreshold laser photocoagulation (micropulse diode laser [MDL] or endpoint laser [EPL]) after providing informed consent according to the routine preoperative care procedure followed in our hospital. All treatments were performed by a single surgeon (K.O.). Because this was a retrospective study, the provision of MDL or EPL treatment could not be controlled.

For MDL treatment, a 577-nm yellow photocoagulation device (Iris Medical OcuLight SLx; IRIDEX Corp, Mountain View, CA, USA) was used in the micropulse operation mode. The laser power for subthreshold treatment of each patient was determined by creating a threshold 
burn using the lowest energy required to create a visible test burn at an area outside the vascular arcade without SRD. The laser parameters were as follows: $5 \%$ duty cycle, $100 \mu \mathrm{m}$, and $200 \mathrm{~ms}$ at titration with the lowest power setting. The laser power was then reduced to $60 \%$ at treatment, using the same parameters. If focal leaking points were clearly visualized, they were treated with a single shot pattern. If SRD persisted or increased, the entire SRD area was treated using a $5 \times 5$ grid pattern with the TxCell mode at $60 \%$ of the energy level to confluent spots (spacing 0 ) in areas up to $500 \mu \mathrm{m}$ from the center of the fovea.

For EPL treatment, a 577-nm diode laser photocoagulation device (PASCAL ${ }^{\circledR}$ yellow; Topcon Corp) was used in the Endpoint Management mode. Similar to the procedure for MDL, we calculated the laser power for subthreshold treatment of each patient by creating a threshold burn using the lowest energy required to create a visible test burn at an area outside the vascular arcade without SRD. Clearly visualized focal leaking points were treated with a single shot pattern. If SRD persisted or increased, laser treatment was applied to the hyperfluorescein SRD areas using a $2 \times 2$ grid pattern (landmark OFF) at $50 \%$ of the energy level to confluent spots at distances of up to $500 \mu \mathrm{m}$ from the center of the fovea. At titration, the laser parameters were $100 \mu \mathrm{m}$ and $15 \mathrm{~ms}$ with the lowest power setting; the laser energy was then reduced to 50\% at treatment by using Endpoint Management.

The Wilcoxon signed-rank test was used to evaluate the collected data. All statistical analyses were performed using SPSS version 21.0J software (SPSS Japan, Tokyo, Japan). A $p$ value of $<0.05$ was considered statistically significant.

Table 1 presents a summary of the characteristics of all eyes. One patient (patient 1) reported a history of intravitreal aflibercept injection for SRD. Visual acuity was maintained in all 5 eyes. The total follow-up duration was 13-26 months. SRF was completely resorbed in 4 eyes ( $80 \%$; patients $1,2,4$, and 5 ) within 4 months after the initial treatment. Two eyes (patients 2 and 5) showed recurrence at 6 months after the initial treatment. Of these, one (patient 5) showed complete SRF reabsorption at 3 months, although recurrence was observed again at 6 months. Finally, the lesion showed spontaneous resorption without additional laser treatment at 7 months. The second eye (patient 2) with recurrence received 2 further EPL treatments, and the SRF was finally resorbed at 26 months. The average time taken for complete SRF resorption in the 4 eyes was 10.25 months after the initial treatment.

Four patients (4 eyes) received subthreshold micropulse laser ( $577 \mathrm{~nm}, 5 \%$ duty cycle, $100 \mu \mathrm{m}, 0.2 \mathrm{~s}$, delivery of $60 \%-80 \%$ of the threshold energy) treatment, while 2 (2 eyes) received Pascal ${ }^{\circledR}$ EPL $(577 \mathrm{~nm}, 100 \mu \mathrm{m}, 0.015 \mathrm{~s}$, delivery of $50 \%-90 \%$ of the threshold energy) treatment. Patient 2 received both MDL and EPL (MDL first, followed by EPL for the second and third treatment sessions). All patients received 1-3 laser treatment sessions (mean: 2.2).

The mean visual acuity ( $\log$ MAR) was $0.28,0.31$, and 0.29 at enrollment and at 1 and 3 months after the initial treatment. No significant difference was observed at 1 and 3 months after the initial treatment. The mean central macular thickness $(\mu \mathrm{m})$ was 355.2, 331.0, and $277 \mu \mathrm{m}$ at enrollment and at 1 and 3 months after the initial treatment, respectively; there was a significant decrease at 3 months $(p=0.043)$. There were no instances of laser scotoma. No laser-induced retinal scarring was observed on color fundus photography at the end of treatment. Two eyes (patients 1 and 3) showed changes in FAF findings at the laser ablation site. One eye (patient 1) underwent MDL twice; hyperfluorescence at the laser ablation site was present at 2 weeks, but this gradually changed to hypofluorescence during the 2 months after the initial treatment. After 4 months, the patient underwent additional laser treatment for persistent SRD. The hyperfluorescent spots reappeared at 1 month and changed to hypofluorescence at 3 months after the second treatment. Another eye (patient 3) underwent MDL twice, although there was no change after the initial treatment. After 3 months, the patient underwent additional laser treatment for persistent SRD; hyperfluorescence was present at

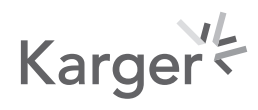




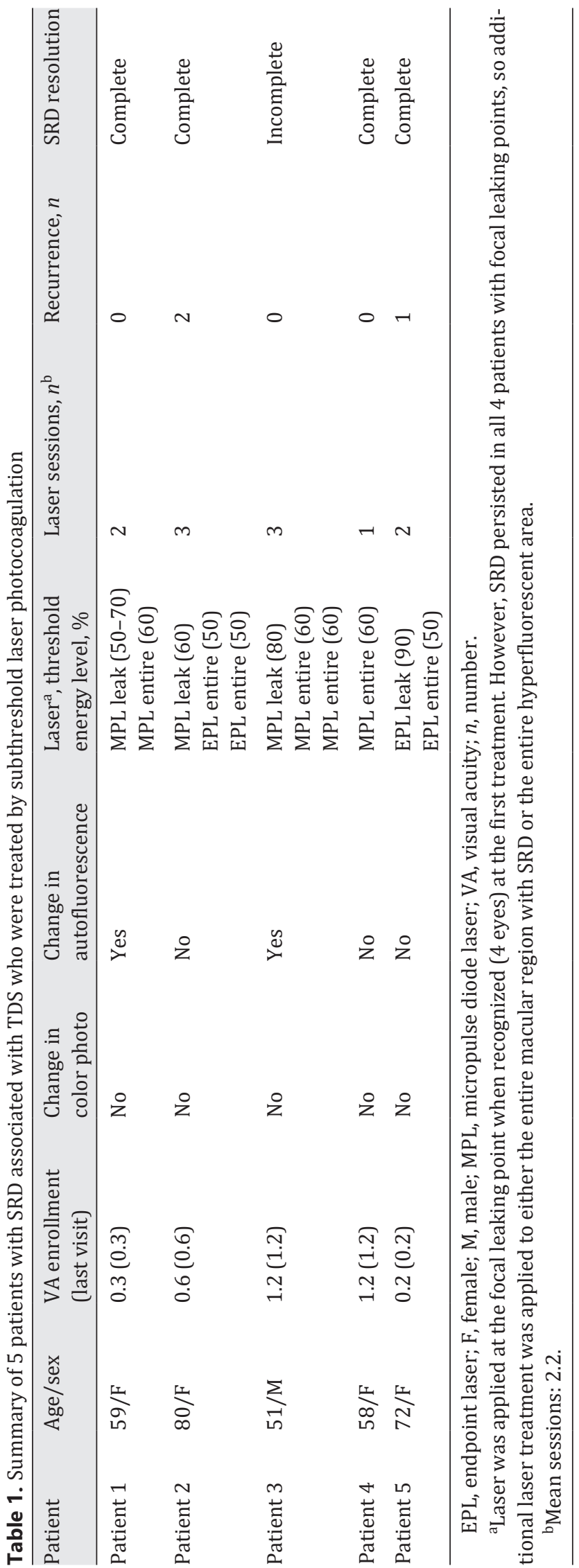

\section{Karger'}



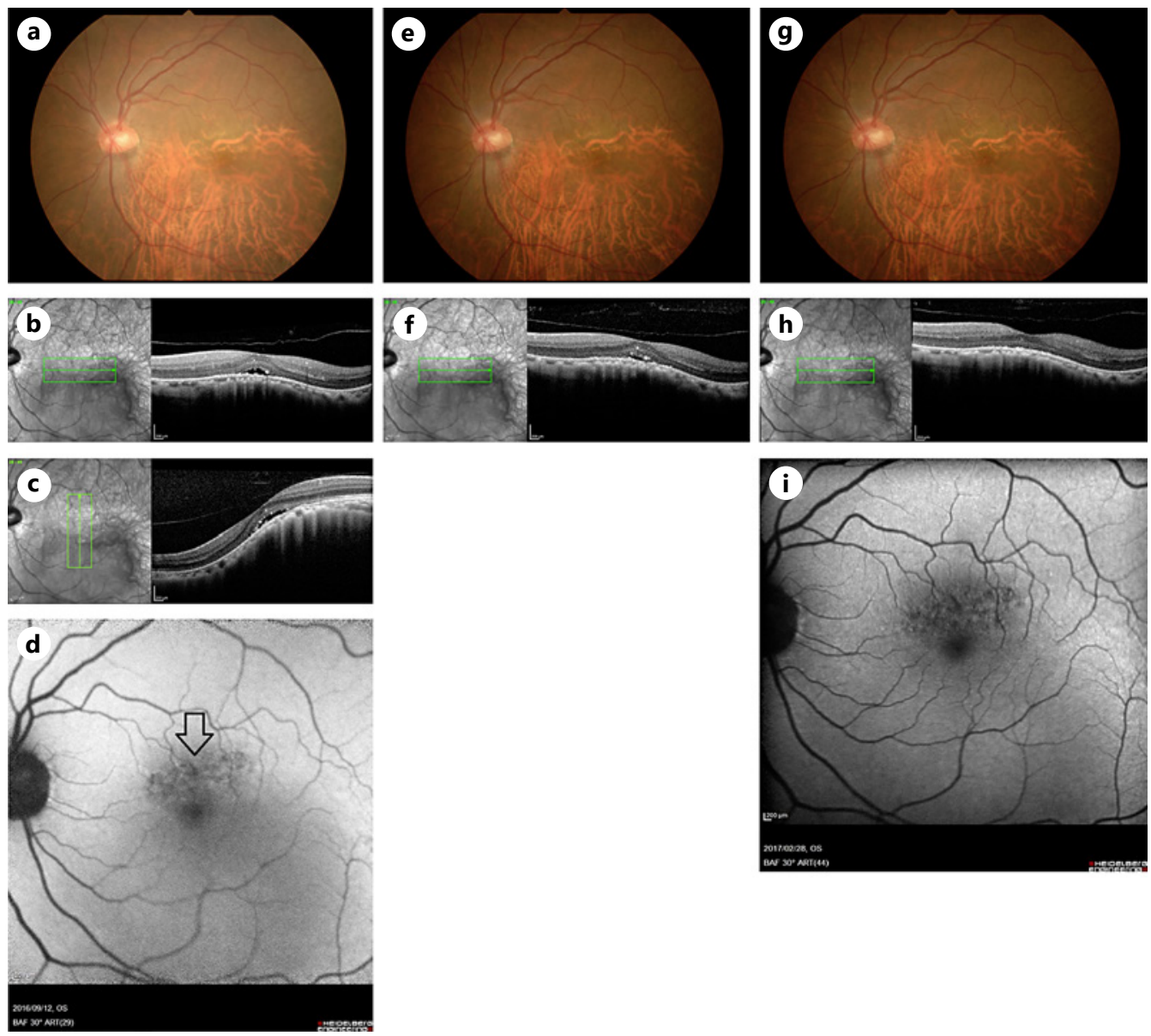

Fig. 1. Clinical course of patient 4 (58-year-old woman), who was successfully treated with subthreshold laser treatment for SRD with TDS. a-d Pretreatment images of patient 4 (58-year-old woman) with TDS. a Fundus color photograph showing TDS and SRD with an inferonasally "tilting" optic disc with inferior staphyloma. b, c Optical coherence tomography showing persistent SRD for $>3$ months. d Fundus autofluorescence showing hypofluorescent spots at the superior macula. e, $\mathbf{f}$ One-month follow-up images of the same patient (patient 4) with TDS and SRD treated by subthreshold laser photocoagulation. e Fundus color photograph showing no change and no obvious laser scarring. f Optical coherence tomography showing a gradual decrease in SRD. g-i Five-month follow-up images of the same patient (patient 4) with TDS and SRD treated by subthreshold laser photocoagulation. g Fundus color photograph showing no change and no obvious laser scarring. $\mathbf{h}$ Optical coherence tomography showing complete resorption of SRD. $\mathbf{i}$ Fundus autofluorescence showing no change.

1 month and changed to hypofluorescence at 3 months after the third treatment. One eye (patient 2) did not undergo FAF at enrollment because the treatment started before FAF was available.

We present the findings for a typical case in Figure 1. A 58-year-old woman (patient 4) with TDS and persistent SRD was treated by MDL. Before treatment, FAF had shown hypofluorescent spots at the superior macula because of RPE degeneration with SRD. Because there were no focal leaking points, MDL was applied to the entire SRD area. SRD gradually decreased at 1 month after treatment and finally disappeared by 5 months. We also present FAF photos showing change after treatment in Figure 2. A 51-year-old man (patient 3) with 

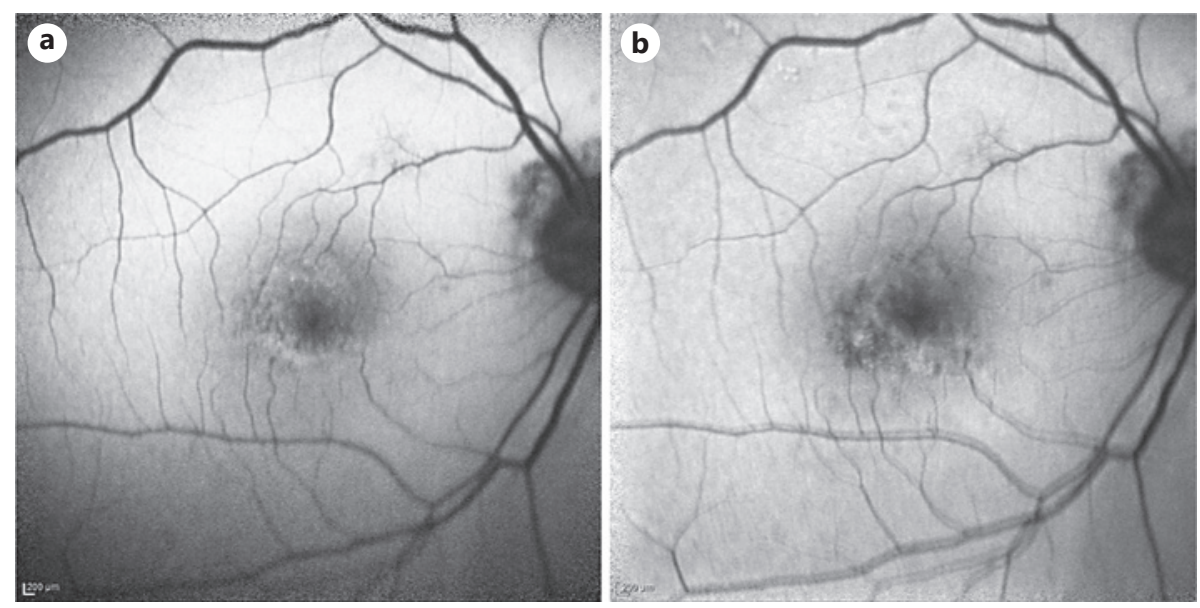

Fig. 2. Pre- and post-treatment images ( 3 months) of patient 3 (51-year-old man) with TDS. a Fundus autofluorescence picture before subthreshold laser photocoagulation. Granular hypofluorescent spots are observed at the foveal area, showing RPE damage. b Fundus autofluorescence picture 3 months after subthreshold laser photocoagulation. Hypofluorescent spots are merged and enlarged especially at the temporal macula.

TDS and persistent SRD was treated by MDL and EPL. Before treatment, the FAF picture had shown hypofluorescent spots at the foveal area because of RPE degeneration with SRD. After treatment, hypofluorescent spots are merged and enlarged.

\section{Discussion/Conclusion}

In this case series, SRF due to TDS was successfully treated with subthreshold laser treatment without damage to the outer retina. The RPE pumping function may be promoted after laser energy-induced remodeling of RPE cells. To our knowledge, this is the first report demonstrating an effective treatment for this condition.

TDS is a congenital anomaly caused by insufficient closure of the embryonic optic fissure. It is characterized by a nasal-to-temporal "tilting" of the optic disc and inferior staphyloma. The mechanism of SRD in patients with TDS is not fully understood. Maruko et al. [2] hypothesized that the choroidal fluid in TDS might not pass through a thickened sclera and may consequently leak into the subretinal space through the degenerated RPE.

The treatment of SRD in patients with TDS has been reported in several previous studies. In 2010, Milani et al. [3] reported a case that received treatment comprising 2 consecutive monthly applications of intravitreal bevacizumab $(1.25 \mathrm{mg})$. In their patients, OCT showed persistence of SRD after 6 months of follow-up. In 2013, Donati et al. [4] reported 3 cases treated with PDT, intravitreal bevacizumab, and combined therapy, respectively. After treatment, there was no change in foveal retinal detachment on OCT images of all eyes. In 2019, Kubota et al. [7] reported 48 eyes which were observed without interventions (16 eyes), treated with PDT (10 eyes), intravitreal bevacizumab (17 eyes), and combined therapy (5 eyes). Compared eyes with or without the interventions, average BCVA were similar between both groups, and on the other hand, last average CRT were significantly decreased in eyes with interventions. The number of eyes without SRD at the last visit was 21 eyes (43.8\%). Although SRD absorption in the present cases might be natural course, 4 eyes (80\%) showed complete resorption of SRF at the end of the treatment. Moreover, these cases had persistent SRD ( $>1$ year) before laser treatment. Although the mechanisms underlying the 
efficacy of subthreshold laser photocoagulation for SRD in patients with TDS are poorly understood, we suggest that alternation of RPE pumping function, which was induced by laser ablation, may play an important role in treatment success.

Conventional thermal laser therapy involves coagulation of the entire retinal layer, including the neurosensory retina, with continuous-wave laser irradiation. Therefore, thermal damage spreads both vertically and horizontally, affecting tissues adjacent to the coagulated RPE layer and resulting in progressive scarring and consequent scotoma [8]. Because of this risk, conventional laser treatment is rarely performed near the fovea. In contrast, subthreshold laser photocoagulation can achieve safer treatment and, consequently, can be performed near the fovea.

Micropulse technology is a unique laser delivery method. Controlled thermal elevation is achieved by "chopping" a continuous-wave beam into an envelope of repetitive short pulses. Because thermal tissue damage is proportional to the exposure time, an ultra-short laser pulse elevates the temperature of only the RPE. Li et al. [9] reported that, after MDL treatment, the protein and messenger RNA expressions of angiogenic stimulators (vascular endothelial growth factor A, transforming growth factor beta, and basic fibroblast growth factor) were significantly decreased. On the other hand, those of the angiogenic inhibitor (pigment epithelium-derived factor) were significantly increased in mouse RPE [9]. Sublethal photostimulation with a micropulse laser on a layer of RPE cells may thus initiate the protein synthesis cascade, leading to activation of the RPE cellular function and resorption of SRD.

In contrast, EPL is a laser treatment system that uses a mathematical model of retinal hyperthermia, based on an Arrhenius Integral, to precisely control power and duration. This enables subthreshold treatment using EPL, which optimizes the therapeutic effect of the laser at less-damaging levels.

Endpoint Management (Topcon Corp) software enables reliable titration to subthreshold treatment. With EPL, the RPE is selectively ablated without significant collateral thermal effects. The therapeutic effects of EPL are indirectly produced by the healing response and the inflammation that occurs in response to RPE coagulation. The cells adjacent to the wound respond by sliding to fill and repair the laser-induced RPE defect. Using scanning electron microscopy, Lavinsky et al. [5] reported recovery of the RPE monolayer in the ablation area 3 days after EPL (50\% of the threshold energy level) treatment in Dutch belted rabbits. Therefore, the newly proliferated RPE may recover the pump function after EPL, leading to SRD resorption.

In our study, 2 eyes showed changes in FAF findings at the laser ablation site after MDL treatment. Although some studies have reported that MDL treatment did not change the FAF findings at the laser site [10] or result in structural changes at the laser ablation site on OCT [11], our data showed changes in FAF findings at the laser site in the 2 cases. Repeated ablation of areas of focal leaking may have caused the changes in FAF findings in our cases. We did not evaluate macular function before and after treatment in our study. However, there were no instances of laser scotoma and no laser-induced retinal scarring on color fundus photography performed at the end of treatment. With respect to macular function after MDL, Hoshikawa et al. [12] reported that the average retinal sensitivity after MDL treatment showed no statistically significant change at 2 weeks. However, long-term observations may be necessary to evaluate the safety of this method.

This study was limited by its single-center retrospective design and the small sample size. A large, multicenter, prospective clinical trial is warranted to confirm the efficacy of subthreshold laser photocoagulation for the treatment of SRD in patients with TDS.

Although the mechanism for fluid resolution remains unknown, we believe that subthreshold laser photocoagulation is an effective and safe treatment modality for SRD in patients with TDS. Thus, the clinical indications of subthreshold laser photocoagulation may be extended to SRD in patients with TDS.

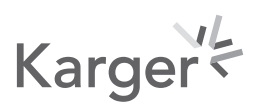




\section{Statement of Ethics}

This retrospective study followed the tenets of the Declaration of Helsinki. The Institutional Review Board at St Luke's International Hospital approved this study (No. 17-R114). At St. Luke's Hospital, the details of all research studies that have passed the institutional review board are posted on the hospital's website, and patients who participate are notified. And only those patients who refuse to participate in the study are asked to state so in writing. Therefore, consent forms are not taken from all patients who participate in the study. This includes informing patients of the option to opt out of the publication of the elements of their medical case included in this article. This consent procedure was reviewed and approved by the Institutional Review Board at St Luke's International Hospital, Approval No. 17-R114.

\section{Conflict of Interest Statement}

The authors have no conflicts of interest to declare.

\section{Funding Sources}

This manuscript did not receive any funding.

\section{Author Contributions}

Y.M. contributed to the design of the work, interpretation of data, writing of manuscript, and creation of figures. K.O. contributed to the design of the work, supervision, and writing of the manuscript. Y.O. contributed to supervision and writing of the manuscript. All authors critically reviewed the manuscript and approved the final version of the manuscript.

\section{Data Availability Statement}

All data generated or analyzed during this study are included in this article. Further enquiries can be directed to the corresponding author.

\section{References}

1 Nakanishi H, Tsujikawa A, Gotoh N, Hayashi H, Iwama D, Tamura H, et al. Macular complications on the border of an inferior staphyloma associated with tilted disc syndrome. Retina. 2008 Nov-Dec;28(10):1493-501.

2 Maruko I, Iida T, Sugano Y, Oyamada H, Sekiryu T. Morphologic choroidal and scleral changes at the macula in tilted disc syndrome with staphyloma using optical coherence tomography. Invest Ophthalmol Vis Sci. 2011 Nov;52(12):8763-8.

3 Milani P, Pece A, Pierro L, Seidenari P, Radice P, Scialdone A. Bevacizumab for macular serous neuroretinal detachment in tilted disk syndrome. J Ophthalmol. 2010 Nov;2010:970580.

4 Donati MC, Miele A, Abbruzzese G, Giuntoli M, Giansanti F, Menchini U. Treatment of macular serous neuroretinal detachment in tilted disk syndrome: report of 3 cases. Eur J Ophthalmol. 2013 Mar-Apr;23(2):26770 .

5 Lavinsky D, Sramek C, Wang J, Huie P, Dalal R, Mandel Y, et al. Subvisible retinal laser therapy: titration algorithm and tissue response. Retina. 2014 Jan;34(1):87-97.

6 Chen SN, Hwang JF, Tseng LF, Lin CJ. Subthreshold diode micropulse photocoagulation for the treatment of chronic central serous chorioretinopathy with juxtafoveal leakage. Ophthalmology. 2008 Dec;115(12):222934. 
Minowa et al.: Subthreshold Laser Treatment for SRD

7 Kubota F, Suetsugu T, Kato A, Gomi F, Takagi S, Kinoshita T, et al. Tilted disc syndrome associated with serous retinal detachment: long-term prognosis. A retrospective multicenter survey. Am J Ophthalmol. 2019 Nov; 207:313-8.

8 Scatz H, Madeira D, McDonald HR, Johnson RN. Progressive enlargement of laser scars following grid laser photocoagulation for diffuse diabetic macular edema. Arch Ophthalmol. 1991 Nov;109(11):1549-51.

9 Li Z, Song Y, Chen X, Chen Z, Ding Q. Biological modulation of mouse RPE cells in response to subthreshold diode micropulse laser treatment. Cell Biochem Biophys. 2015 Nov;73(2):545-52.

10 Ohkoshi K, Tsuiki E, Kitaoka T, Yamaguchi T. Visualization of subthreshold micropulse diode laser photocoagulation by scanning laser ophthalmoscopy in the retro mode. Am J Ophthalmol. 2010 Dec;150(6):856-62.

11 Inagaki K, Ohkoshi K, Ohde S. Spectral-domain optical coherence tomography imaging of retinal changes after conventional multicolor laser, subthreshold micropulse diode laser, or pattern scanning laser therapy in Japanese with macular edema. Retina. 2012 Sep;32(8):1592-600.

12 Hoshikawa Y, Ohkoshi K, Yamaguchi T. [Retinal sensitivity following subthreshold diode laser micropulse photocoagulation for diabetic macular edema]. Nippon Ganka Gakkai Zasshi. 2011 Jan;115(1):13-9. 